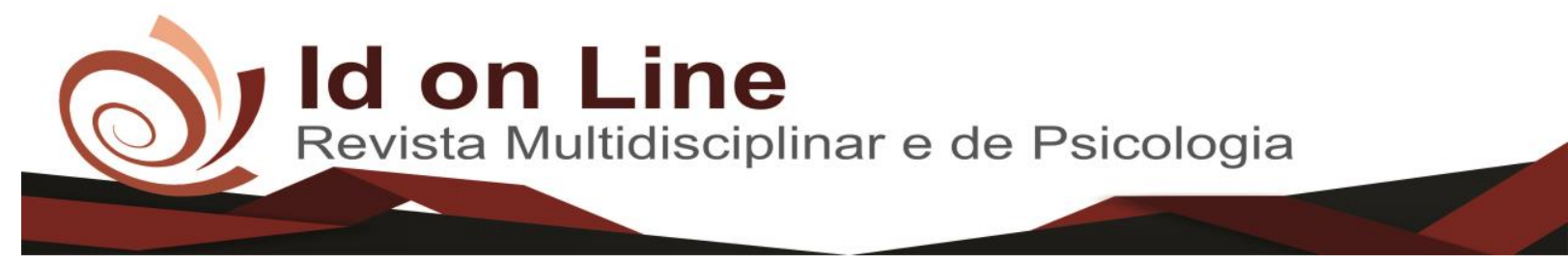

Artigo

\title{
Habilidades Sociais no Contexto da Psicologia Organizacional e do Trabalho
}

\author{
Jedídja Hadassa de Santana Varela ${ }^{1}$, Ahiram Brunni Cartaxo de Castro ${ }^{2}$, Lydia Maria Pinto Brito ${ }^{3}$, \\ Pablo Marlon Medeiros da Silva $a^{4}$ Arthur William Pereira da Silva ${ }^{5}$
}

\begin{abstract}
Resumo: Este artigo investiga a utilização do conceito de Habilidades Sociais (HS) no contexto da Psicologia Organizacional e do Trabalho (POT). Inicialmente, foram pontuados aspectos históricos do mundo do trabalho e da POT como campo de estudo e, a partir disso, discute-se o conceito de HS. A questão de pesquisa é: qual o estado da discussão da aplicação de HS para a POT? A pesquisa se justifica, pois o trabalho como um fator constitutivo do homem, fomentou pesquisas e discussões no tempo que marcam a relevância das HS para o trabalhador; a POT, por sua vez, se destaca como um campo de investigação dos mais profícuos da área. Nesta perspectiva, as HS se apresentam como um tópico de estudo passível de diálogo com a POT, pois, esse conceito está sendo utilizado, mesmo se tratando de uma aplicação tradicionalmente feita na psicologia clínica e escolar. O objetivo da pesquisa é investigar a aplicação do conceito de HS para a POT. Utilizou-se de uma análise documental de natureza exploratória que tem quadro de referência na compreensão. Percebeu-se que, a aproximação das HS com a POT pode se consolidar como prática no trabalho. No entanto, requer esclarecimentos, devido ao percurso teórico do conceito de HS e pela facilidade com que os profissionais utilizam o treinamento de habilidades sociais na área organizacional. A POT tem seus desafios e também é diversa/abrangente, portanto, deve-se atentar ao fato de um novo conceito que possa somar a estes desafios.
\end{abstract}

Palavras-chave: Habilidades Sociais. Psicologia Organizacional e do Trabalho. Treinamento de Habilidades Sociais.

\section{Social Skills in the Context of Organizational and Work Psychology}

\begin{abstract}
This article investigates the use of the concept of Social Skills (SS) in the context of Organizational Psychology and Work (OPW). Initially, historical aspects of the world of work and OPW were analyzed as a field of study and, from this, the concept of HS is discussed. The question of research is: what is the state of the discussion of the application of HS to OPW? The research is justified, since work as a constitutive factor of man, fomented researches and discussions in the time that mark the relevance of SS for the worker; the OPW, in turn,
\end{abstract}

\footnotetext{
${ }^{1}$ Especialista em Psicologia Organizacional e do Trabalho e em Terapia Cognitivo Comportamental pela Universidade Federal do Rio Grande do Norte (UFRN, RN). Graduada em Psicologia pela Universidade Potiguar (UNP, RN). jhadassa2@ gmail.com;

2 Doutorando em Administração e Mestre em Administração pela Universidade Potiguar (UNP, RN), especialista Gestão de Pessoas pela Universidade Federal do Rio Grande do Norte (UFRN, RN). Graduado em Administração pela Universidade Potiguar (UNP, RN). Administrador no Instituto Federal de Educação, Ciência e Tecnologia do Rio Grande do Norte (IFRN, RN). brunnicastro@ hotmail.com;

${ }^{3}$ Doutorado em Educação (2004) pela Universidade Federal do Ceará - UFC. Mestrado em Sociologia (1998). Graduação em Serviço social (1974) e especialização em Teoria e Prática de Serviço Social (1980) pela Universidade Estadual do Ceará - UECE. Professora Titular de PósGraduação em Administração (Doutorado e Mestrado) da Universidade Potiguar (UNP, RN). lydiampbrito@yahoo.com.br;

4 Doutorando em Administração e Mestre em Administração pela Universidade Potiguar (UNP). Graduado em Administração pela Universidade Federal Rural do Semi-Árido (UFERSA).pablo_marlon17@ @otmail.com;

${ }^{5}$ Doutorando em Administração pela Universidade Potiguar (UNP), Mestrando em Administração pela Universidade Federal da Paraíba (UFPB), Mestre em Ambiente, Tecnologia e Sociedade pela Universidade Federal Rural do Semi-Árido (UFERSA) e Graduado em Administração pela Universidade Potiguar (UNP). arthurwilliamadm@ hotmail.com.
} 
stands out as a field of investigation of the most proficient in the area. In this perspective, SS are presented as a topic of study that can be discussed with OPW, since this concept is being used, even if it is an application traditionally made in clinical and school psychology. The objective of the research is to investigate the application of the HS concept to OPW. A documentary analysis of an exploratory nature was used that has frame of reference in the understanding. It was noticed that the approximation of the SS with the OPW can be consolidated as practice in the work. However, it requires clarification, due to the theoretical course of the SS concept and the ease with which the professionals use the social skills training in the organizational area. The OPW has its challenges and is also diverse / comprehensive, so we must pay attention to the fact that a new concept that can add to these challenges.

Keywords: Social skills; Organizational and Work Psychology; Social Skills Training.

\section{Introdução}

$\mathrm{Na}$ contemporaneidade, os estudos sobre o trabalho são cada vez mais presentes, recriados e discutidos. O mundo do trabalho pode ser caracterizado de várias formas, pois o sentido da palavra trabalho foi construído por muitos significados desde as civilizações antigas até os atuais modelos de bem-estar. Nesse contexto, a área de Psicologia Organizacional e do Trabalho (POT) se apresenta como um dos campos construtores do diálogo sobre o trabalho junto das organizações.

Diante disso tem-se o seguinte questionamento: qual o estado da discussão da aplicação de Habilidades Sociais para a Psicologia Organizacional e do Trabalho?

A pesquisa se justifica, pois, o trabalho como um fator constitutivo do homem, vem fomentando pesquisas e discussões que assinalam a clara relevância das habilidades sociais para a trajetória do trabalhador na chamada modernidade. A área da Psicologia Organizacional e do Trabalho (POT), por sua vez, destaca-se como um campo de investigação dos mais profícuos no que se refere a esse tema. Nesta perspectiva, as Habilidades Sociais (HS) apresentam-se como um tópico de estudo passível de diálogo com a POT, já que, esse conceito vem sendo utilizado em organizações (MORENO-JIMÉNEZ et al., 2014; VELOSO-BESIO et al., 2015; SEKIGUCHI; HOSOMI, 2017; YUN et al., 2017), mesmo se tratando de uma aplicação tradicionalmente feita na área da Psicologia Clínica e Escolar (VERA et al., 2017; POULOU, 2017; MORRIS; MCGUIRE; WALKER, 2017; TUCKER, 2017). 
Por isso, pontua-se a necessidade do resgate teórico das HS, do levantamento das aplicações do Treinamento de Habilidades Sociais (THS) nas organizações e com trabalhadores, e da continuada investigação de estratégias de trabalho na POT. O fato de que a Psicologia Organizacional vem colocando desafios aos profissionais da área, e que o conceito de HS começa a se estabelecer com efetividade nas organizações torna-se um importante aspecto acadêmico para a investigação.

Outrossim, conforme Sánchez e Escribano (1999); Inocente e Guimarães (2004); Bolsoni-Silva et al. (2006); Moreno-Jiménez et al., 2014, a literatura brasileira e internacional sobre o HS na área organizacional ainda não é tão vasta. As HS tem sido aplicadas preferencialmente em atividades de Treinamento de Habilidades Sociais (THS) para a formação profissional pré-laboral com indivíduos incapacitados e em situações de desvantagem sociais (SÁNCHEZ; ESCRIBANO, 1999; INOCENTE; GUIMARÃES, 2004; BOLSONI-SILVA et al., 2006; BANDEIRA et al., 2009; FISHER; MORIN, 2017; MORRIS; MCGUIRE; WALKER, 2017; PEREIRA-LIMA; LOUREIRO, 2017; LAUGEN et al., 2017) e para o desenvolvimento de lideranças de equipes de alto desempenho (FAGOULIS; PHILLIPS, 2011; HURRELL; SCHOLARIOSPUKK, 2014; CUADRA-PERALTA et al., 2017). Desta feita seriam importantes investigações que trouxessem mudanças positivas para a realidade no contexto do trabalho e do trabalhador.

Portanto, o objetivo da pesquisa é investigar a aplicação do conceito de Habilidades Sociais para Psicologia Organizacional e do Trabalho.

Além da introdução, tem-se os procedimentos metodológicos, e em seguida a pesquisa explora no referencial teórico uma breve discussão sócio-histórica sobre o homem e o trabalho, já que o movimento de construção da POT se inicia com o surgimento do capitalismo e, do mesmo modo, as definições das HS são também ligadas a contextos sociais, mutáveis. Por fim, são apresentadas as considerações finais e as referências.

\section{Procedimentos Metodológicos}

Quanto aos meios tem-se uma análise documental. Conforme Cellard (2008), o documento em questão consiste em todo texto escrito, manuscrito ou impresso, registrado em 
papel. Mas precisamente, as fontes primárias ou secundárias, que por definição, são exploradas - e não criadas - no contex to de um procedimento de pesquisa.

Utilizou-se da análise de documentos secundários públicos não arquivados, sendo: manuscritos publicados em periódicos internacionais disponibilizados na base de dados Scopus no período de 2000 a 2017 sob os termos social skills or social skills training, sendo consideradas suas escritas no singular e no plural nos títulos dos artigos. E, livros publicados sobre a temática sem período de busca definido.

A escolha do corpus de análise se deu à luz do questionamento inicial da pesquisa. Os procedimentos adotados para a análise documental garantem a procedência e a qualidade dos documentos analisados. (CELLARD, 2008).

Os documentos foram previamente identificados e foi realizado um inventário e uma seleção da informação disponível por área do conhecimento, quando foram consideradas as áreas de Psicologia, Administração e Ciências Sociais, com documentos na língua inglesa, espanhola e em português, evitando-se a precipitação sobre o primeiro bloco de documentos obtidos.

Quanto a natureza, trata-se de uma pesquisa exploratória. Conforme Acevedo e Nohara (2006, p. 46), “O principal objetivo da pesquisa exploratória é proporcionar maior compreensão do fenômeno que está sendo investigado, permitindo assim que o pesquisador delineie de forma mais precisa o problema".

De acordo com Mattar (2001); Alexandre (2003); Gil (2006) a pesquisa exploratória, visa prover ao pesquisador maior esclarecimento, modificar conceitos e ideias através da pesquisa documental. Compatível com o objetivo da pesquisa.

Portanto, o quadro de referencia da pesquisa repousa na compreensão (BRUYNE; HERMAN; SCHOUTHEETE, 1991).

\section{Referencial Teórico}

Fazendo-se a distinção do pior arquiteto para a melhor aranha, Marx (2013), ilustrou e destacou a intencionalidade humana para diferenciar o trabalho humano em relação ao trabalho animal, focando, assim, na intermediação da cultura sobre o homem. Ele entendia que: “[...] o 
trabalho deveria ser humanizador, não-alienado, digno, que garantisse ao ser humano a satisfação de suas necessidades, racional (com uma divisão baseada em critério de igualdade entre os homens) e que se constituísse na principal força na vida dos indivíduos”.

Esta intermediação cultural traz à tona uma sequência de aprendizados incorporados pelo homem que, mesmo ao longo de tantos anos de evolução, nenhum animal pôde alcançar, e é nesta complexidade que se insere o trabalho e suas relações.

Esta perspectiva, junto aos pensamentos que surgiram sobre o trabalho desde as civilizações antigas, até os modelos de bem-estar e trabalho das sociedades de hoje, fizeram emergir as delimitações do que Borges e Yamamoto (2004) buscam explorar como o mundo do trabalho.

Borges e Yamamoto (2004) colocam que, no trabalho o que distingue mais claramente a transformação da matéria natural é o objetivo, a intenção que direciona propositadamente a ação do homem. Portanto, não há como abdicar das possibilidades intencionais ou cognitivas do homem, pois isso descaracterizaria o próprio sentido do trabalho. É a partir da perspectiva de um trabalho humano e intencional, diferente do trabalho animal; também atravessado pelas culturas; que grande parte dos que fazem a Psicologia Organizacional do Trabalho fundamentam seus estudos. Neste sentido, o foco é a discussão quanto aos aspectos influenciadores da condição humana em seu trabalho.

O mundo do trabalho pode ser caracterizado de várias formas. O próprio sentido da palavra trabalho permeia muitos significados que variam em épocas e situações. $\mathrm{O}$ trabalho pode referir-se às relações de poder (SILVEIRA, 2005; MATOS; MESQUITA; MACHADO, 2017), à natureza da atividade que é feita, ao emprego, a questões de formalidade e informalidade, à complexidade, à qualificação, entre outros, dessa forma, as pesquisas sobre o significado do trabalho, indicam que a variedade de significados guarda entre si relações, mas também, muitas contradições (BORGES; YAMAMOTO, 2004).

Considerando o aspecto econômico, o trabalho também encontra definições variadas, por exemplo, um aspecto comum na literatura é a discussão do contrato de trabalho que diferencia o trabalho do emprego. Para Borges e Yamamoto (2004), a indistinção destes dois conceitos dificultava a discussão sobre o papel do trabalhador no século XX e sobre a importância que as pessoas atribuem ao trabalho. 
O significado do trabalho segue as condições sócio-históricas em que cada pessoa vive, "e é neste sentido que falar sobre mundo do trabalho torna-se apenas um recorte da diversidade neste contexto”. (BORGES; YAMAMOTO, 2004, p. 27).

Os autores dividiram didaticamente dimensões relacionadas ao trabalho. São elas:

a) dimensão concreta: se refere ás condições materiais e/ou ambientais para realização do trabalho;

b) dimensão gerencial: refere-se ao modelo de gerir, planejar, organizar, dirigir e controlar o exercício das funções;

c) dimensão sócio-econômica: abrange a articulação entre o modo de realizar o trabalho e as estruturas sociais, econômicas e políticas em plano macro da sociedade, incluindo aí aspectos dinâmicos econômico-sociais;

d) dimensão ideológica: refere-se ao discurso elaborado e articulado sobre o trabalho, justificando principalmente as relações de poder na sociedade; e,

e) dimensão simbólica: consiste nos aspectos subjetivos da relação de cada indivíduo com seu trabalho. Estas seriam as dimensões constituintes do mundo do trabalho. (BORGES; YAMAMOTO, 2004).

Sócio-historicamente, para entender as transformações no mundo do trabalho revolvese ao capitalismo industrial e, chegando ao processo de industrialização no Brasil, Borges e Yamamoto (2004, p. 42) explicam que, no país, "a absorção do modelo (de desenvolvimento industrial) foi intermediada pelo ‘capitalismo tardio' e pelas desigualdades entre regiões e setores econômicos". Disto pode ser entendido que, diferentemente da Europa aqui as mudanças não ocorreram de forma gradativa, ao longo dos anos e suas construções históricas, mas sim de forma imperativa.

O mundo do trabalho, ao longo destas modificações relatadas anteriormente, vem se reconfigurando, e junto a ele a POT também, por isso, os conhecimentos envolvidos estão sendo construídos, numa constante releitura das realidades e das demandas surgidas nas organizações e na sociedade.

Defendendo-se um equilíbrio na construção da identidade do homem diante do trabalho nas organizações e dos diversos aspectos que influenciam o exercício da subjetividade humana, articulando-se também o contex to social e histórico do trabalho; é que se constituiu o caminho da POT (CODO; SORATTO; VASQUES-MENEZES, 2004). 
Discorrer sobre o trabalho e suas várias fases implica também em entender as concepções de sujeito e de mundo prevalentes nos períodos antigos e contemporâneos. Neste sentido, o significado do trabalho vai tomando rumos diferentes, e são usadas técnicas e construtos, dos quais a POT se apropriou, enquanto recursos para a ampliação do campo de investigação e clarificação de novos objetos de estudo. Relacionar as HS à POT seria uma proposta inicial para contribuir com este conhecimento.

\title{
Trabalho e Psicologia Organizacional
}

Malvezzi (2004, p. 13) explica que:

\begin{abstract}
Estudando problemas tais como o desempenho, a saúde do trabalhador, seus padrões de qualidade de vida, o impacto do emprego, das condições de trabalho sobre a vida humana, tanto sob o ponto de vista de atividade pessoal como de atividade institucionalizada, a POT criou uma massa de conhecimentos que, embora controvertida em vários aspectos (Setffy e Grimes, 1992; Prilleltensky, 1994), tais como sua base epistemológica e os valores a ela associados, fez-se presente em toda a história da gestão de pessoas, através de vários tipos de contribuição.
\end{abstract}

Em entrevista à Revista Diálogos (2008), Antonio Virgílio elucida que a necessidade de uma visão de integralidade do ser humano é missão da POT, diante das multiplicidades de papéis que o trabalho, como instituição, tem sob a vida das pessoas.

Malvezzi (2004) explica que a investigação produzida sobre tal tema tem se focado nos aspectos da eficiência do desempenho para a produção (dimensão regulatória), investigando problemas humanos significativos, porém, fora de seus contextos; e, respondidas às demandas sensíveis das elites, mais do que mergulhado na compreensão da relação homem-trabalho, na construção dos sujeitos e no empenho pela diminuição das desigualdades (dimensão emancipatória).

As mudanças sócio-econômicas que ocorreram durante o movimento industrial inglês gerou mudanças na economia mundial, no homem e em seu comportamento no âmbito organizacional. É neste contexto que a área da POT inicia-se como campo mais efetivo de atuação. O comportamento organizacional recebe estas interferências sócio-econômicas e a POT tem buscado atualizar e reconhecer estes processos com vastas indicações teóricas. Neste sentido, alguns estudiosos destacam-se por delinearem o campo da POT e embasarem as investigações feitas nesta área do conhecimento. 
Dentre os autores, destacam-se: Mustemberg, que se interessou pela seleção de pessoal e o uso de testes psicológicos para maior ajuste das pessoas aos cargos num contexto industrial; Patrizi e Kraepelim, que estudaram a fadiga e a carga de trabalho; Taylor, precursor de uma administração científica; Gilbreth e Frank, psicólogos, que desenvolverem o que veio a ser chamado de estudo de tempos e movimentos; Mayo, que, com a experiência de Hawthorne, inaugurou o movimento das Relações Humanas no campo da administração industrial; Maslow, que desenvolveu o estudo da pirâmide das necessidades humanas; McGregor com suas proposições sobre a teoria $\mathrm{X}$ (referindo-se às abordagens organizacionais tradicionais) e teoria $Y$ (referindo-se às abordagens organizacionais emergentes) para as pessoas nas empresas. Entre outros tantos nomes, tais autores até ultrapassaram o conhecimento chamado de psicológico e foram atravessados pela demanda da realidade de suas épocas (TAMAYO, TRÓCCOLI, 2002).

Num processo contínuo de construção e desconstrução, do tipo evolutivo, as conjunturas econômicas e sociais vão interferindo nas relações de trabalho e modificando o homem, não só enquanto trabalhador, mas também enquanto sujeito deste processo. As organizações, neste sentido, sentem claramente estas mudanças, pois conforme colocam Zanelli e Bastos (2004, p. 468):

\begin{abstract}
A segunda metade do século XX foi marcada por intenso desenvolvimento tecnológico e movimentos que culminaram no que tem sido comum chamar de novas práticas de gestão: por exemplo, a gestão da qualidade total, a cultura de aprendizagem, o empowerment, o trabalho em equipes, as células de produção, a produção just-in-time entre outras.
\end{abstract}

No Brasil, ao final da década de 1980, segundo Zanelli e Bastos (2004) os psicólogos tinham seu espaço nas organizações vinculado às ações de recrutamento e seleção. Para os autores, os psicólogos não conseguiam ter uma visão clara do produto de seu trabalho, não havia resoluções efetivas para as demandas existentes, e sim ações pontuais e limitadas.

Quando questionado sobre qual a perspectiva da evolução da POT no Brasil, pela revista Diálogos, Malvezzi (2004, p. 29) explica que:

[...] a POT é um campo especializado de conhecimentos, situado na interface entre a saúde, a administração, a política, a engenharia e a moral, produzidas pelo trabalho institucionalizado, através do qual a instrumentalização da produção e a qualidade de vida possam ser desenvolvidas a serviço da vida individual e coletiva.

Na perspectiva das fontes de emprego (empresas, empresários, redes governamentais) e estudiosos do trabalho, as pessoas destacam-se como um aspecto diferenciador, muito mais que os recursos materiais. A ideia geral para isto é a de que o recurso material, ou mesmo 
estratégico, é passível de ser reconhecido e copiado pela concorrência. No entanto, o recurso humano dentro dos processos mais diversos, faz a diferença pela existência inegável das formas de expressão da singularidade humana, não podendo ser copiado. Nesta visão, no entanto, ocorre uma supervalorização do papel das pessoas; algumas vezes, em detrimento das condições que elas têm para o trabalho.

Zanelli e Bastos (2004, p.13) explica que "[...] a aplicação dos recursos no trabalho é balizada por valores, relações de poder, significados e conhecimentos que constituem a base de sua institucionalização". O autor afirma que o estudo do trabalho tornou-se uma área transdisciplinar que facilitou o diálogo entre as ciências sociais, mas que há lacunas nestas relações institucionalizantes.

Ainda segundo Zanelli e Bastos (2004, p. 14) uma das situações colocadas para a missão da POT é a de que "[...] as pessoas têm sido solicitadas a aprender habilidades em tempo mais curto que sua condição humana permite, ou solicitadas a alterar suas identidades sem que isso faça parte de seus planos de vida".

Os modelos da Gestão por Competências, Organizações de Aprendizagem e Gestão do Conhecimento, por exemplo, surgidos por volta dos anos 90, trouxeram a concepção de que as organizações que apresentavam lucro, que se expandiam no mercado e se projetavam para o futuro, tinham "aprendido a aprender" a se adaptarem com rapidez às demandas do mercado e apresentavam como características marcantes a gestão de sua competência essencial, a gestão do conhecimento necessário para projetar a empresa para o futuro e a tentativa de mudar/ revitalizar sua cultura organizacional (SENGE, 2012).

Nesse contexto, o ponto principal desses novos modelos é o indivíduo, ou seja, as pessoas passam a figurar como os principais ativos estratégicos - as pessoas e suas competências, já que o conhecimento reside nas pessoas e é na sua interação, ou seja, através das habilidades das pessoas (inteligência, sensibilidade, discernimento, criatividade e capacidade de inovação), que o conhecimento é disseminado provocando-se a aprendizagem organizacional.

Esses trabalhadores foram denominados por Drucker (1999) de "trabalhadores do conhecimento".

Bons trabalhadores do conhecimento deveriam, portanto, ter uma combinação de competências hard (conhecimento estruturado, qualificações técnicas e experiência 
profissional) e de atributos soft (um claro senso dos aspectos culturais, políticos e pessoais do conhecimento) (DAVENPOR; PRUSAK,1997; MACEDO et al., 2017).

Segundo Del Prette e Del Prette (1999; 2009), a competência técnica usualmente faz parte dos objetivos educativos de cursos profissionalizantes e dos treinamentos que ocorrem no âmbito das organizações, a exemplo das universidades corporativas. No entanto, a competência interpessoal pouco é relacionada como objetivo de formação profissional ocorrendo como um subproduto do processo educativo, por vezes no currículo oculto das formações.

Por participar historicamente do desenvolvimento da concepção de trabalho, no sentido de que se entende que as mudanças aconteceram não somente, na matéria, ou nas formas de trabalho, mas também na vida psíquica, econômica, social, cultural e política do ser humano, é que a POT discute estratégias na gestão dos negócios, e por assim dizer, no contexto do trabalho.

Del Prette e Del Prette (2009) ainda acrescenta que, os conhecimentos produzidos pela POT tornaram viáveis tanto a utilização de tecnologias sofisticadas, por parte de grupos de trabalho constituídos por pessoas simples, como a formação e a adaptação de equipes interdependentes, aptas para o desempenho eficaz de múltiplas atividades sofisticadas, como se observa nos trabalhos dos astronautas. Além disso, problematizam colocando que "[...] um dos mais significativos desafios do trabalhador é um enfrentamento rotineiro das descontinuidades que o obrigam a um contínuo ajustamento, para o qual the falta sensibilidade ou apoio institucional." (p. 15).

Questões que se asseveram no atual ambiente social e mercadológico, marcado pela complexidade, dinamismo, contrariedade, imprevistos, incertezas, redes, liquidez, medo, sociosfera e ciberesfera (BAUMAN, 2004, 2010; ABRANCHES, 2017).

Para Del Prette e Del Prette (2009), embora se reconheça a dificuldade de pesquisa nesta população (trabalhadores), deve se considerar, também, sua importância, tanto na formação de agentes socialmente competentes, como por meio de uma interação produtiva, beneficiar os indivíduos por eles atendidos. Por outro lado, o amplo conhecimento de habilidades sociais, como requisito da competência e do sucesso profissional. Os autores supõem a investigação mais detalhada desta temática, com outros grupos de formação ou atuação em diferentes áreas.

Além disto, segundo Del Prette e Del Prette (2009) expõe a existência de novos paradigmas organizacionais que enfatizam a importância da qualidade nas relações interpessoais, devendo haver uma multiespecialização e "a valorização do trabalho em equipe, intuição, criatividade e autonomia na tomada de decisões", entre outros aspectos, gerando uma 
demanda por certas habilidades (coordenação de grupo, liderança de equipes, manejo de estresse e de conflitos interpessoais, etc.) (p. 57). Como forma de enfrentamento do atual cenário em que o trabalho está inserido.

Os autores destacam que o trabalho em pequenos grupos mostra a necessidade de habilidades de supervisão e monitoramento de tarefas e interações relacionadas ao processo produtivo que, para ocorrerem adequadamente, exigem competência em requisitos como os de observar, ouvir, dar feedback, descrever, pedir mudança de comportamento, perguntar e responder perguntas entre outras.

Inocente e Guimarães (2004, p. 129) afirma que "paradoxalmente, o local de trabalho é um dos ambientes nos quais mais ocorrem conflitos interpessoais, sendo que a habilidade para resolvê-los é pouco considerada".

Conforme Inocente e Guimarães (2004, p. 136), notaram que “[...] os trabalhadores que apresentam um repertório comportamental habilidoso possuem muito mais chances de sucesso que os que apresentam comportamento inassertivo ou agressivo, mesmo apresentando nível intelectual semelhante".

Revelando-se em nível organizacional a existência da aplicação de Habilidades Sociais associadas ao trabalho.

\section{Habilidades Sociais e Psicologia Organizacional e do Trabalho}

Enfatiza-se aqui a utilização dos estudos e práticas relacionados às Habilidades Sociais no campo da POT, levando-se em conta o fato de que o Treinamento de Habilidades Sociais (THS) é uma das técnicas da terapia comportamental mais utilizadas no atendimento clínico, escolar e no contexto organizacional e do trabalho, devido a sua eficácia. (INOCENTE; GUIMARÃES, 2004; BOLSONI-SILVA et al., 2006; WHITE; KEONIG; SCAHILLDEL PRETTE, 2007; DEL PRETTE, 2008; BANDEIRA et al., 2009; CIA; BARHAM, 2009; PUKK; GAFFNEY, 2015).

Sabendo disto, observa-se a aplicação das técnicas, com pouca proximidade teórica, no que se refere ao campo de estudo da POT, e, a partir de então buscam-se diálogos possíveis.

O conceito de Habilidades Sociais tem como plano de fundo o desempenho social. É um conceito que atravessa mais de um modelo teórico explicativo, e que foi, e ainda é 
transformado pelo tempo, por buscar estabelecer explicações a comportamentos e situações mutáveis, com uma diversidade que vem construindo um vasto campo de estudo.

Assim como a área de Treinamento de Habilidades Sociais (THS), o conceito de Habilidades Sociais (HS), segundo Del Prette e Del Prette (1999, p. 24), “[...] constitui um campo de investigação e de aplicação do conhecimento psicológico sobre o desempenho social". Os autores explicam que "[...] trata-se de um campo com questões teóricas e práticas cujos significados e desdobramentos são bem mais amplos e complexos do que pode sugerir um exame superficial."

Olaz (2009, p. 110) coloca que:

[...] sem dúvida, os avanços na investigação não foram acompanhados por um desenvolvimento teórico que permita sistematizar estes resultados e, na atualidade, coexistem diferentes abordagens orientadas para a pesquisa e a intervenção sobre o comportamento social que utilizam uma terminologia diversificada para referir-se ao mesmo fenômeno.

Apesar da tentativa de clarificação do conceito por autores como Del Prette e Del Prette (2009), há uma multiplicidade de conceitos referentes a fenômenos sociais semelhantes. Por exemplo, às nomenclaturas dadas à relacionamento interpessoal, competência social, relações humanas, competências, além do que, a nomenclatura do conceito HS, historicamente também passou por modificações. Ou seja, observa-se um percurso histórico conceitual que, para chegar ao que é hoje citado pelos autores, foi se modificando e estas modificações incluem também a nomenclatura dada.

Assim, é possível afirmar que ao se falar em HS deve-se reconhecer que se trata, ainda hoje, de um conceito em discussão, mas que este possui suas origens em aspectos cognitivos, comportamentais e sociais anteriores; e talvez por se tratar de um recorte que envolve também o aspecto social e cultural, torna-se ainda pouco delineado.

Del Prette e Del Prette (1999) e parceiros, que possuem vasta publicação nesta área (FREITAS; DEL PRETTE, 2011; FREITAS; DEL PRETTE, 2015; LOPES et al., 2015; LEME et al., 2016; FRANÇA-FREITAS; DEL PRETTE; DEL PRETTE, 2017; QUELUZ et al., 2017), fundamentam suas explanações no que se inicia com Salter, em sua obra Conditioned Reflex Therapy, de 1949. Neste caso, o treinamento assertivo, atrelado a movimentos paralelos aos estudos sobre desempenho social teriam sido os antecessores do que se estabeleceu como a aplicação do conceito de Habilidades Sociais, que é o Treinamento de Habilidades Sociais. 
Wolpe, em 1958, propagou as ideias de Salter utilizando-se do "[...] termo comportamento assertivo como sendo uma habilidade social importante para o relacionamento interpessoal" (INOCENTE; GUIMARÃES, 2004, p. 131).

Segundo Del Prette e Del Prette (1999, p. 27):

Quase concomitantemente ao nascimento do Treinamento Assertivo nos Estados Unidos, um novo movimento se iniciava na Inglaterra, mais precisamente na Universidade de Oxford, com Argyle e outros pesquisadores a ele associados. (Argyle, 1967/1994, 1975; Argyle, Trower \& Bryant, 1974; Trower, Bryant \&Argyle, 1978) disseminando o uso do termo 'habilidades sociais'.

Estes autores explicam que Argyle iniciou seus estudos na década de 60 aplicando o conceito de habilidades às interações homem-máquina, a partir da investigação de atividades que remetiam ao processamento de informações e a aspectos tais como a percepção, decodificações e a resolução de problemas (DEL PRETTE; DEL PRETTE, 1999).

Explorando a área de Treinamento de Habilidades Sociais, Del Prette e Del Prette (1999), consideram que há cinco modelos conceituais: o modelo da assertividade, o modelo da percepção social, o modelo da aprendizagem social, o modelo cognitivo e o modelo da teoria de papéis.

Del Prette e Del Prette (1999, p. 33) colocam que, considerando-se o modelo cognitivo “[...] A avaliação e a intervenção sobre esses processos (cognitivos) é amplamente enfatizada no THS".

Nota-se que a combinação dos modelos-explicativos permite uma exploração mais abrangente dos possíveis fatores e das alternativas de intervenção sobre os problemas de déficits de habilidades (DEL PRETTE; DEL PRETTE, 2009).

"Atualmente, a tendência é utilizar o termo habilidade social por ser mais abrangente e aplicável em diferentes contextos, como na escola, na clínica e no trabalho." (INOCENTE; GUIMARÃES, 2004, p. 132).

Nesse sentido, Del Prette e Del Prette (1999) conceituam habilidades sociais como "habilidades para organizar cognições e comportamentos em um curso de ação integrada, dirigida para objetivos sociais ou interpessoais culturalmente aceitáveis" (p. 127). Para os autores a perspectiva que remete a cultura, ainda deixa lacunas neste conceito.

$\mathrm{Na}$ análise das habilidades sociais e da competência social, considerando que este conceito é um construto avaliativo (que geraria um juízo de valor) e não descritivo, como o de HS, é possível observar três dimensões importantes: a pessoal, a situacional e a cultural; Tais 
dimensões se interpenetram e estão sempre presentes nas interações sociais, podendo-se dizer que o comportamento social depende dessa combinação. (DEL PRETTE; DEL PRETTE, 1999).

As várias definições para Habilidades Sociais, segundo Inocente e Guimarães (2004) aparecem paralelamente aos estudos do comportamento assertivo. No entanto, este conceito diferencia-se do conceito de habilidades sociais quando a assertividade refere-se "à habilidade de influenciar os outros através da expressão apropriada de emoções." (INOCENTE; GUMARÃES, 2004, p. 132). As HS, por sua vez, referem-se, mais abrangentemente, a um repertório de respostas sociais, tanto verbais quanto não verbais.

Para Inocente e Guimarães (2004); Vera et al. (2017); Poulou (2017), o comportamento socialmente habilidoso está relacionado a um conjunto de comportamentos adequados a situação, que expressam emoções, sentimentos, opiniões, direitos; respeitando os outros, prevenindo e resolvendo possíveis problemas.

Já Inocente e Guimarães (2004); Gadaire, Marshall e Brissett (2017), enfatizam o reforçamento positivo nas situações interpessoais com resposta positiva para auto-estima do indivíduo, definindo, a partir disto, a habilidade social.

Gresham (2009, p. 20) demonstra que: "Merrell e Gimpel (1998) identificaram pelo menos quinze definições de habilidades sociais que surgiram na literatura especializada". O autor coloca o entendimento de HS como uma classe de respostas comportamentais, considerando a perspectiva da Análise Aplicada do Comportamento. As habilidades sociais poderiam ser comportamentos aprendidos e socialmente aceitáveis que permitiriam interagir efetivamente, evitando interações sociais negativas.

Gresham (2009, p. 20) ainda coloca que “[...] as habilidades sociais são competências que (a) facilitam a iniciação e manutenção de relacionamentos sociais positivos, (b) contribuem para a aceitação por colegas e (c) resultam em ajustamento escolar satisfatório."

Para Caballo, Irurtia e Salazar (2009, p. 68), há dois autores mais representativos com relação a um referencial cognitivo sobre a HS. Estes autores, McFall e Trower, estabeleceram um marco fundamentalmente cognitivo para as HS. "Do ponto de vista cognitivo, uma resposta socialmente habilidosa é o resultado final de uma cadeia de comportamentos [...]" (CABALLO; IRURTIA; SALAZAR, 2009, p. 69).

Considerando este modelo cognitivo, Caballo (1996); Caballo, Irurtia e Salazar (2009) destacam as competências cognitivas, tentando agrupar os processos cognitivos estudados na 
literatura sobre as HS. A competência cognitiva se referiria à capacidade adquirida de "construir ativamente uma multiplicidade de comportamentos potenciais, habilidosos, adaptativos, que têm consequências [...]” (p. 72). Os autores expõem grandes diferenças na amplitude e qualidade dos padrões cognitivos das diferentes pessoas e elencam elementos para observação dessas competências, que seriam: conhecimento do comportamento habilidoso, conhecimento dos costumes sociais, conhecimento dos diferentes sinais de resposta, saber colocar-se no lugar da outra pessoa, e capacidade de solução de problemas.

De acordo com Olaz (2009), os modelos explicativos para as HS têm sido denominados “interativos" por considerarem a realidade das variáveis ambientais e características pessoais, o comportamento, e as interações, entre esses aspectos.

Olaz (2009); Poulou (2017); Salavera, Usán e Jarie (2017), explicam que o fato dos estudos terem destacado a especificidade situacional das HS e do comportamento social em geral, contribuiu para a diferenciação do conceito de HS de outros surgidos sob o modelo de atributo, como por exemplo, o conceito de Inteligência Emocional.

Além deste aspecto, na perspectiva interacionista, a Teoria Social Cognitiva "[...] foi um dos sistemas de maior importância na explicação do comportamento, e seus princípios sustentam em grande medida as estratégias de avaliação e intervenção em HS” (OLAZ, 2009, p. 112).

Por fim, cita-se os autores Del Prette e Del Prette (1999, p. 29), que podem desmembrar momentaneamente estas interferências conceituais, e possuem literatura correspondente ao assunto, quando explicam que: "as habilidades sociais correspondem a um universo mais abrangente das relações interpessoais e se estendem para além da assertividade, incluindo as habilidades de comunicação, de resolução de problemas, de cooperação[...]".

\section{Considerações Provisórias}

Concluindo-se este recorte, pontua-se a necessidade do resgate teórico das HS, do levantamento das aplicações do THS nas organizações e com trabalhadores e da continuada investigação de estratégias de trabalho na POT. Deve-se, ainda, atentar para o fato de que a Psicologia Organizacional vem colocando desafios aos profissionais da área, e que o conceito de HS começa a se estabelecer com efetividade nas organizações. 
Por outro lado, percebeu-se que a literatura sobre o Habilidades Sociais na área organizacional ainda está em construção e o tema tem sido aplicado preferencialmente em atividades de Treinamento de Habilidades Sociais, ou seja, associado no contexto do trabalho ao treinamento para a formação profissional pré-laboral com indivíduos incapacitados e em situações de desvantagem sociais, e para o desenvolvimento de lideranças de equipe de alto desempenho, pois se trata de um conceito em discussão, mas que este possui suas origens em aspectos cognitivos, comportamentais e sociais. Quanto aos aspectos social e cultural, a temática torna-se ainda pouco delineada.

Surgem assim, lacunas diante dos objetivos ideológicos ou até profissionais para a POT, que seriam importantes investigações futuras para trazer mudanças positivas para a realidade no contexto do trabalho. Portanto, o conceito de HS pode contribuir de forma efetiva com estas mudanças? Considerando isto, ainda há bastante para se pesquisar.

\section{Referências}

ABRANCHES, Sérgio. A era do imprevisto: a grande transição do Século XXI. São Paulo: Companhia das Letras, 2017.

ACEVEDO, C. R.; NOHARA, J. J. Monografia no curso de administração: guia completo de conteúdo e forma. 2. ed. São Paulo: Atlas, 2006.

ALEXANDRE, Mário J. O. A construção do trabalho científico: um guia para projetos, pesquisas e relatórios científicos. Rio de Janeiro: Forense Universitária, 2003.

BAUMAN, Zygmunt. Capitalismo Parasitário: e outros temas contemporâneos. Rio de Janeiro: Jorge Zahar Editora, 2010.

Editora, 2004.

Amor líquido: sobre a fragilidade dos laços humanos. Rio de Janeiro: Jorge Zahar

BANDEIRA, M. et al. Validação das Escalas de Habilidades Sociais, Comportamentos Problemáticos e Competência Acadêmica (SSRS-BR) para o Ensino Fundamental. Revista Psicologia: Teoria e Pesquisa, Abr-Jun, 2009, Vol. 25, n. 2, pp. 271-282.

BOLSONI-SILVA, A. T. et al. Habilidades Sociais no Brasil: Uma análise dos estudos publicados em periódicos. São Paulo: Casa do Psicólogo, 2006. 
BORGES, L. O.; YAMAMOTO, O.H. O Mundo do Trabalho. In: ZANELLI, J.C..BORGES ANDRADE, J.E. e BASTOS, A.V.B. Psicologia organizações e trabalho no Brasil. Porto Alegre; Artmed, 2004.

BRUYNE, Paul de; HERMAN, Jacques; SCHOUTHEETE, M. de. Dinâmica da pesquisa em ciências sociais: os pólos da prática metodológica. Rio de Janeiro: F. Alves, 1991.

CABAllo, V. E. Manual de Técnicas de Terapia e Modificação do Comportamento. Ed. Santos, 1996.

; IRURTIA, M. J.; SALAZAR, I. C. Abordagem Cognitiva na avaliação e intervenção sobre habilidades sociais. In: DEL PRETTE, Z. A.P.; DEL PRETTE, A. Psicologia das Habilidades Sociais: diversidade teórica e suas implicações. Vozes. Petrópolis, 2009.

CELLARD, A. A análise documental. In POUPART, J.; DESLAURIERS, J-P.; GROULX, L-H.; LAPERRIÈRE, A.; MAYER, R.; PIRES, A.P. A pesquisa qualitativa - Enfoques epistemológicos e metodológicos. Petrópolis: Vozes, 2008. (p. 295-316).

CIA, Fabiana; BARHAM, Elizabeth J. Repertório de habilidades Sociais, problemas de comportamento, autoconceito e desempenho acadêmico de crianças no início da escolarização. Revista Estudos de Psicologia, Vol. 26, pp. 45-55, 2009.

CODO, W; SORATTO, L.; VASQUES-MENEZES, I. Saúde mental e trabalho. In: ZANELLI, J.C.BORGES ANDRADE, J.E. e BASTOS, A.V.B. Psicologia organizações e trabalho no Brasil. Porto Alegre; Artmed, 2004.

CUADRA-PERALTA, Alejandro Adrian et al. Intervention for supervisors, based on social skills and leadership, in order to improve organizational climate perception and organizational performance outcomes. Journal of Organizational Change Management, v. 30, n. 2, p. 281$292,2017$.

DAVENPORT, Thomas; PRUSAK, Lawrence. Conhecimento empresarial: como as organizações gerenciam o seu capital intelectual. Rio de Janeiro: Elsevier, 1998.

DEL PRETTE, A.; DEL PRETTE, Z. A. P. Psicologia das Habilidades Sociais: Diversidade teórica e suas implicações. Petrópolis, RJ: Vozes, 2009.

Um sistema de categorias de habilidades sociais educativas. Revista Paidéia, Vol. 18, pag. 517-530, 2008.

Vozes, 1999.

Psicologia das Habilidades Sociais: terapia, educação e trabalho. Petrópolis, RJ,

DRUCKER, Peter F. Desafios gerenciais para o século XXI. Pioneira, 1999.

FAGOULIS, Iosif; PHILLIPS, Niki. Social skills for successful career development. Review of European Studies, v. 3, n. 1, p. 85, 2011. 
FISHER, Marisa H.; MORIN, Lindsay. Addressing social skills deficits in adults with Williams syndrome. Research in Developmental Disabilities, v. 71, p. 77-87, 2017.

FRANÇA-FREITAS, Maria L. P. de; DEL PRETTE, Almir; DEL PRETTE, Zilda A. P. Habilidades Sociais e Bem-Estar Subjetivo de Crianças Dotadas e Talentosas. Psico-USF, v. 22, n. 1, p. 1-12, 2017.

FREITAS, Lucas C.; DEL PRETTE, Zilda A. P. Social Skills Rating System-Brazilian version: New exploratory and confirmatory factorial analyses. Avances en Psicología Latinoamericana, v. 33, n. 1, p. 135-156, 2015.

FREITAS, Lucas C.; DEL PRETTE, Zilda A. P. Validade de critério do sistema de avaliação de habilidades sociais (SSRS-BR). Psicologia: Reflexão e Critica, 23(3), p. 430-439, 2011.

GADAIRE, Dana M.; MARSHALL, Genevieve; BRISSETT, Elanor. Differential reinforcement of low rate responding in social skills training. Learning and Motivation, v. 60, p. 34-40, 2017.

GIL, A. Carlos. Método e técnicas de pesquisa social. 5. ed. São Paulo: Atlas, 2006.

GRESHAM, F.M. Análise do Comportamento aplicada às Habilidades Sociais. In: DEL PRETTE, Z. A.P.; DEL PRETTE, A. Psicologia das Habilidades Sociais: diversidade teórica e suas implicações. Vozes. Petrópolis, 2009.

HURRELL, Scott A.; SCHOLARIOS, Dora. "The People Make the Brand" Reducing Social Skills Gaps Through Person-Brand Fit and Human Resource Management Practices. Journal of Service Research, v. 17, n. 1, p. 54-67, 2014.

INOCENTE, N. J. ; GUIMARÃES, L. A. M. Habilidades Sociais no Trabalho. In GUIMARÃES, L. A. M.; GRUBITS, S. Série Saúde Mental e Trabalho. Casa do Psicólogo. São Paulo, 2004.

LAUGEN, Nina J. et al. Social skills in preschool children with unilateral and mild bilateral hearing loss. Deafness \& Education International, v. 19, n. 2, p. 54-62, 2017.

LEME, Vanessa B. R. et al. Habilidades sociais e o modelo bioecológico do desenvolvimento humano: análise e perspectivas. Psicologia \& Sociedade, v. 28, n. 1, 2016.

LOPES, Daniele C. et al. Social skills: A key factor for engineering students to develop interpersonal skills. International journal of Engineering Education, v. 31, n. 1, p. 405-413, 2015.

MACEDO, Valéria et al. Tipologia do trabalhador do conhecimento: papéis e processos. Perspectivas em Gestão \& Conhecimento, João Pessoa, v. 7, Número Especial, p. 94-108, mar. 2017.

MALVEZZI, S. Prefácio. In ZANELLI, J.C..BORGES ANDRADE, J.E. e BASTOS, A.V.B. Psicologia organizações e trabalho no Brasil. Porto Alegre; Artmed, 2004. 
MARX, K. O capital: crítica da economia política. São Paulo: Boitempo, 2013.

MATOS, Fátima R. N.; MESQUITA, Rafael F. de; MACHADO, Diego de Q. (Org.) Estudos observacionais em linguagem fílmica: ética e poder. Curitiba: Editora Prismas, 2017.

MATTAR, F. N. Pesquisa de Marketing: metodologia, planejamento, execução e análise. 3. ed. São Paulo: Atlas, 2001.

MORENO-JIMÉNEZ, Bernardo et al. Habilidades sociales para las nuevas organizaciones. Psicología Conductual, v. 22, n. 3, p. 585, 2014.

MORRIS, Thomas; MCGUIRE, Margit; WALKER, Bridget. Integrating social studies and social skills for students with emotional and behavioral disabilities: A mixed methods study. The Journal of Social Studies Research, 2017.

OLAZ, F. O. Contribuições da Teoria Social Cognitiva de Bandura para o Treinamento de Habilidades Sociais. In: DEL PRETTE, Z. A.P.; DEL PRETTE, A. Psicologia das Habilidades Sociais: diversidade teórica e suas implicações. Vozes. Petrópolis, 2009.

PEREIRA-LIMA, Karina; LOUREIRO, Sonia Regina. Associations between social skills and burnout dimensions in medical residents. Estudos de Psicologia (Campinas), v. 34, n. 2, p. 281-292, 2017.

POULOU, Maria S. The Relation of Teachers' Emotional Intelligence and Students' Social Skills to Students' Emotional and Behavioral Difficulties: A Study of Preschool Teachers' Perceptions. Early Education and Development, p. 1-15, 2017.

PUKK, Härenstam K.; GAFFNEY, D. Cognitive and social skills are necessary for safe teamwork. Lakartidningen, v. 112, 2015.

QUELUZ, Ferraresi R. et al. Inventário de Habilidades Sociais para Cuidadores de Idosos (IHSCI): evidências de validade. Avaliação Psicológica, v. 16, n. 1, 2017.

REVISTA CIÊNCIA E PROFISSÃO. Diálogos. Psicologia Organizacional e do Trabalho, no 5. Brasília: Dezembro/2007. p. 28-31.

SALAVERA, Carlos; USÁN, Pablo; JARIE, Laurane. Emotional intelligence and social skills on self-efficacy in Secondary Education students. Are there gender differences?. Journal of Adolescence, v. 60, p. 39-46, 2017.

SÁNCHEZ, A. V.; ESCRIBANO, E. A. Medição do autoconceito. Bauru, SP: Edusc, 1999.

SENGE, Peter R. (2012). A quinta Disciplina: arte e prática da organização que Aprende. São Paulo: Best Seller.

SILVEIRA, Rafael A. da. Michel Foucault, poder e análise das organizações. FGV Editora, 2005 . 
SEKIGUCHI, Tomoki; LI, Jie; HOSOMI, Masaki. Predicting Job Crafting From the Socially Embedded Perspective: The Interactive Effect of Job Autonomy, Social Skill, and Employee Status. The Journal of Applied Behavioral Science, v. 53, n. 4, p. 470-497, 2017.

TAMAYO, M. R.; TRÓCCOLI, B. T. Exaustão Emocional: Relações com a percepção de suporte organizacional e com as estratégias de coping no trabalho. Revista Estudos de Psicologia. Brasília, 2002.

TUCKER, Catherine et al. Enhancing social-emotional skills in at-risk preschool students through Theraplay based groups: The Sunshine Circle Model. International Journal of Play Therapy, v. 26, n. 4, p. 185, 2017.

VELOSO-BESIO, Constanza et al. Workers training: impact of a program based on positive psychology and social skills, on life satisfaction, job satisfaction and organizational climate. INTERCIENCIA, v. 40, n. 11, p. 736-743, 2015.

VERA, Elizabeth M. et al. Parental Messages, School Belonging, Social Skills, and Personal Control as Predictors of Bullying in Ethnic Minority Adolescents. School Mental Health, v. 9, n. 4, p. 347-359, 2017.

WHITE, Susan Williams; KEONIG, Kathleen; SCAHILL, Lawrence. Social skills development in children with autism spectrum disorders: A review of the intervention research. Journal of autism and developmental disorders, v. 37, n. 10, p. 1858-1868, 2007.

YUN, Yang-Joong et al. Social skills as a moderator between R\&D personnel's knowledge sharing and job performance. Journal of Managerial Psychology, v. 32, n. 5, p. 387-400, 2017.

ZANELLI, J. C.; BASTOS, A. V. B. Inserção Profissional do Psicólogo em Organizações e no Trabalho. In: ZANELLI, J.C..BORGES ANDRADE, J.E. e BASTOS, A.V.B. Psicologia organizações e trabalho no Brasil. Porto Alegre; Artmed, 2004.

\section{Como citar este artigo (Formato ABNT):}

VARELA, Jedídja Hadassa de S.; CASTRO, Ahiram Brunni C. de; BRITO, Lydia M. P.; SILVA, Pablo Marlon M. da; SILVA, Arthur William P. da. Habilidades Sociais no Contexto da Psicologia Organizacional e do Trabalho. Id on Line Rev.Mult. Psic., 2018, vol.12, n.40, p.764-783. ISSN: 1981-1179.

Recebido: 16/01/2018

Aceito 21/05/2018 